\title{
ChemComm
}

\section{Self-organized cobalt fluoride nanochannel layers used as a pseudocapacitor material $\dagger$}

Cite this: Chem. Commun., 2014 50, 7067

Received 14th February 2014 Accepted 6th May 2014

DOI: $10.1039 / c 4 c c 01195 c$

www.rsc.org/chemcomm

\author{
Chong-Yong Lee, ${ }^{a}$ Zixue Su, ${ }^{a}$ Kiyoung Lee, ${ }^{a}$ Hiroaki Tsuchiya ${ }^{b}$ and Patrik Schmuki ${ }^{a}$
}

\begin{abstract}
Aligned $\mathrm{CoF}_{2}$ nanochannel layers have been formed by self-ordering electrochemical anodization. In voltammograms these layers provide multiple oxidation states, an almost ideal rectangular pseudocapacitive behavior, a high specific capacitance and good capacitance retention. These layers may thus be promising for supercapacitor applications.
\end{abstract}

Supercapacitors have matured significantly over the last decade and emerged with the potential to facilitate major advances in energy storage. ${ }^{1,2}$ The approaches of the technology can be classified into two categories, namely electrical double layer capacitors (EDLCs) and pseudocapacitors. ${ }^{3}$ In contrast to EDLCs, where the charge is stored electrostatically, pseudocapacitors store charge through faradaic reactions, i.e., they involve the transfer of charge between electrode and electrolyte and thus a redox state switching at the electrode materials. In comparison to EDLCs, pseudocapacitive materials achieve a substantially higher specific capacitance than EDLCs but usually suffer from an inferior rate performance, low utilization yield of active materials, poor cycling stability, and a poor electrical conductivity of the electrode materials. ${ }^{3}$ The most common pseudocapacitors are based on suitable transition metal oxides (hydroxides) and conductive polymers.

To enhance the performance of pseudocapacitors, new active materials and construction of pseudocapacitance geometries with large specific surface area, porosity, and suitable electronic conductivity are desired. Hence, nanostructuring efforts target the fabrication of nanoporous morphological structures (e.g., nanotubes, ${ }^{4-6}$ nanowires ${ }^{7-9}$ ) that exhibit a higher specific capacitance than their bulk material, and also possess a higher stability. Furthermore, one-dimensional nanostructured materials could provide short diffusion lengths to ions and thus lead to higher charge-discharge rates.

\footnotetext{
${ }^{a}$ Department of Materials Science and Engineering, University of Erlangen-Nuremberg, Martensstrasse 7, D-91058 Erlangen, Germany.E-mail: schmuki@ww.uni-erlangen.de

${ }^{b}$ Division of Materials and Manufacturing Science, Graduate School of Engineering, Osaka University, 2-1 Yamada-oka, Suita, Osaka 565-0871, Japan

$\dagger$ Electronic supplementary information (ESI) available: Experimental details, SEM images, cyclic voltammogram. See DOI: 10.1039/c4cc01195c
}

Among all the transition metal oxides, hydrous $\mathrm{RuO}_{2}$ represents the benchmark system with excellent reversibility and ideal solid-state faradaic reactions involving several oxidation states. ${ }^{10,11}$ However, the material is relatively expensive for the realization of commercial applications. Spinel cobalt oxides provide a reliable alternative due to their low cost and the high theoretical capacitance $\left(3560 \mathrm{~F} \mathrm{~g}^{-1}\right){ }^{12}$ Furthermore, several approaches are available to easily form porous surface morphologies and thus to provide the accessibility of electrolyte ions throughout the structure. Recently, we demonstrated that by optimized anodization of Co, a highly aligned nanochannel structure can be formed, which by a suitable heat-treatment can be readily converted to $\mathrm{Co}_{3} \mathrm{O}_{4} \cdot{ }^{13}$ These channel layers can be grown to several 10 micrometers in thickness on the Co substrate with an ordered pore arrangement. In the present work, we show that anodic $\mathrm{CoF}_{2}$ layers can directly be exploited as a pseudocapacitor material with excellent performance. To the best of our knowledge, this is the first demonstration of a fluoride based cobalt compound that exhibits a well-defined two consecutive electron transfer process with excellent pseudocapacitative properties.

The formation of the cobalt fluoride nanoporous layer is, as other cases of self-organizing anodization, based on establishing a suitable formation/dissolution equilibrium. ${ }^{13-17}$ We found that anodization in a mixed ethylene glycol and glycerol solvent (volume ratio of 1 to 3 ) serves the purpose well. Fig. 1a shows the top view of a Co sheet after anodizing at $50 \mathrm{~V}$ at $0{ }^{\circ} \mathrm{C}$ for $8 \mathrm{~h}$ that leads to an ordered pore structure with pore diameters in the range of 50 to $100 \mathrm{~nm}$ (Fig. 1a) and a layer thickness of $\sim 7 \mu \mathrm{m}$ (Fig. 1b).

Transmission electron microscopy (TEM) images of the $\mathrm{CoF}_{2}$ structure in Fig. 1c show that the nanoporous structures have a wall thickness of $\sim 70 \mathrm{~nm}$. A high degree of crystallinity of $\mathrm{CoF}_{2}$ is evident from the selected-area electron diffraction (SAED) pattern in Fig. 1d. The diffraction patterns can be entirely indexed as $\mathrm{CoF}_{2}$. The XRD pattern of the layer (Fig. 1e) shows a preferential 101 orientation, with peaks that match well with the commercially available $\mathrm{CoF}_{2}$. Fig. 1 f shows the X-ray photoelectron spectroscopy (XPS) depth profile analyses of the as 

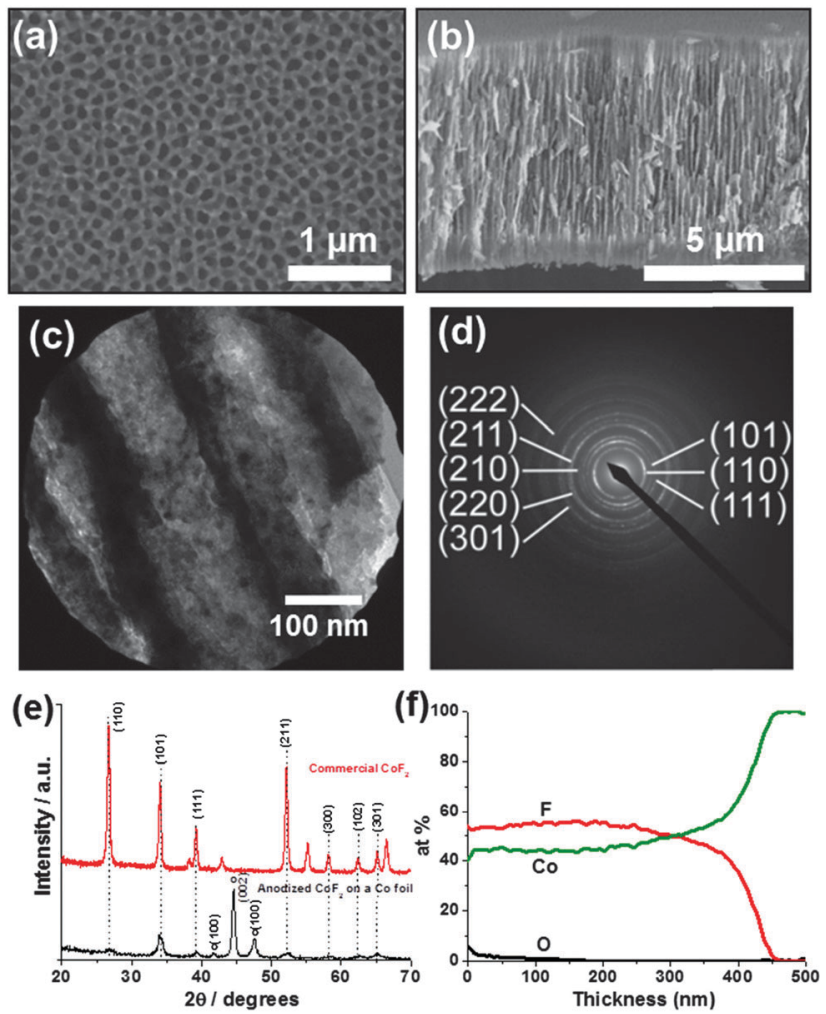

Fig. 1 Top (a) and side (b) views of SEM images of the nanoporous anodic $\mathrm{CoF}_{2}$ layer grown with thickness of $7 \mu \mathrm{m}$. (c) TEM bright-field image of the anodic $\mathrm{CoF}_{2}$ nanoporous structure and (d) the corresponding selective area electron diffraction pattern. (e) XRD of the as prepared sample in comparison to the commercially available $\mathrm{CoF}_{2}$ sample. (f) XPS depth profiles of $\mathrm{Co}, \mathrm{O}$, and $\mathrm{F}$ of the as anodized $\mathrm{CoF}_{2}$ sample with a layer thickness of $\sim 400 \mathrm{~nm}$.

formed $\mathrm{CoF}_{2}$ with a layer thickness of $\sim 400 \mathrm{~nm}$. As expected, $\mathrm{Co}$ and $\mathrm{F}$ are dominant in the layer, but an appreciable amount of $\mathrm{O}$ is detected that is mainly present in the top layer with a concentration that diminishes across the layer. It can be deduced that the layer is mainly present as $\mathrm{CoF}_{2}$ that contains a small percentage of cobalt-oxy-fluoride, Co-O-F, in an amorphous form.

To evaluate the electrochemical properties of the $\mathrm{CoF}_{2}$ layers, electrochemical measurements were performed in a three-electrode electrochemical configuration with a platinum counter electrode and an $\mathrm{Ag} / \mathrm{AgCl}(3 \mathrm{M} \mathrm{KCl})$ reference electrode in $1.0 \mathrm{M} \mathrm{KOH}$ solution. SEM examination of the electrodes after potential cycling (Fig. 2a-d) shows, in comparison to Fig. 1a and b, an alteration of the surface morphology. Higher magnification images in Fig. $2 b$ and $\mathrm{d}$ for the top and cross-sections show platelet formation on the surface (see also additional SEM images in Fig. S1, ESI $\dagger$ ). If $\mathrm{Co}_{3} \mathrm{O}_{4}$ structures are cycled in $\mathrm{KOH}$ solution, the surface morphology remains unaffected as shown in Fig. S2 (ESI $\dagger$ ). It is important to note, however, that although such a surface alteration for the $\mathrm{CoF}_{2}$ structures upon potential cycling in $\mathrm{KOH}$ solution was observed, the nanochannel morphology remains well intact.

The cyclic voltammograms of the $\mathrm{CoF}_{2}$ and the corresponding $\mathrm{Co}_{3} \mathrm{O}_{4}$ (converted by thermal annealing in air at $350{ }^{\circ} \mathrm{C}$ for $30 \mathrm{~min}$ ) as shown in Fig. 2e reveal the differences in the voltammograms where the latter exhibits a more rectangular shape. Essentially, two
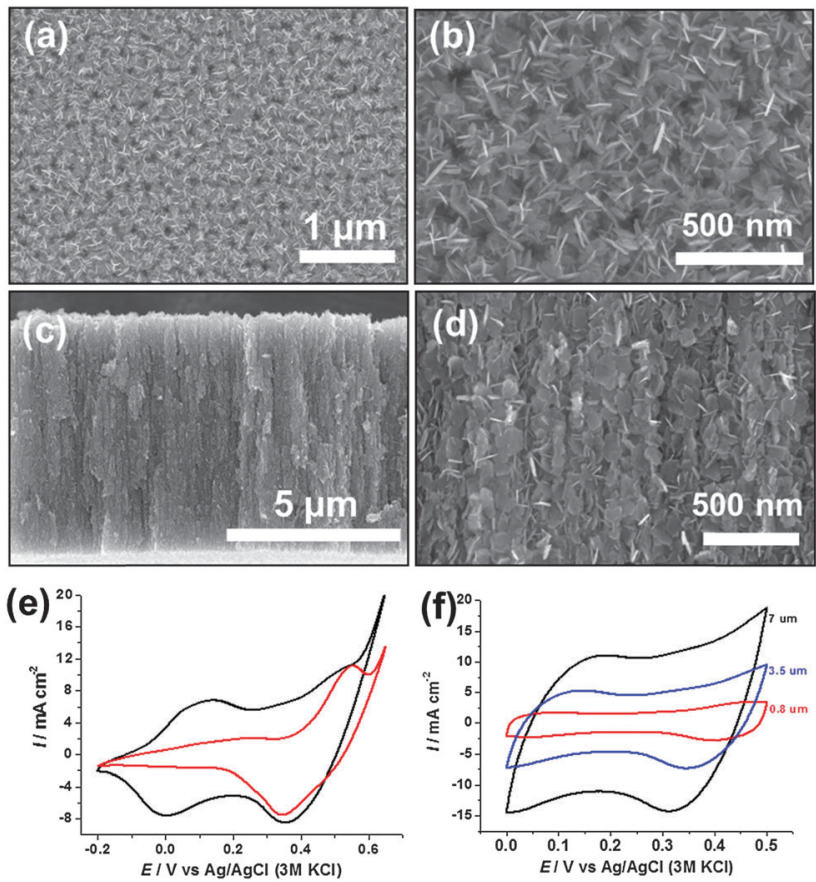

Fig. 2 Top (a, b) and cross (c, d) section views of low and high magnification SEM images of the $\mathrm{CoF}_{2}$ layers after potential cycling in $1 \mathrm{M} \mathrm{KOH}$ solution. (e) Comparison of the cyclic voltammograms of the $\mathrm{CoF}_{2}$ layer with $\mathrm{CO}_{3} \mathrm{O}_{4}$ layer $\left(\mathrm{CoF}_{2}\right.$ after heat-treatment at $300{ }^{\circ} \mathrm{C}$ for $\left.30 \mathrm{~min}\right)$ of thickness of $3.5 \mu \mathrm{m}$ in $1 \mathrm{M} \mathrm{KOH}$ solution. (f) Cyclic voltammograms of the $\mathrm{CoF}_{2}$ samples with various layer thicknesses. Scan rate $=50 \mathrm{mV} \mathrm{s}^{-1}$.

distinct redox peaks become apparent for the $\mathrm{CoF}_{2}$ sample while only one single redox peak in the case where $\mathrm{Co}_{3} \mathrm{O}_{4}$ is present. This supports the hypotheses that the initial state of the $\mathrm{CoF}_{2}$ sample is indeed in the oxidation state of two and then undergoes two sequential electron transfer steps from $\mathrm{Co}$ (II) to $\mathrm{Co}$ (III) and $\mathrm{Co}(\mathrm{III})$ to $\mathrm{Co}(\mathrm{IV})$. Examination of the scan rate dependence (see Fig. S3a, ESI $\dagger$ ) further reveals that the as synthesized $\mathrm{CoF}_{2}$ possesses faster redox kinetics than $\mathrm{Co}_{3} \mathrm{O}_{4}$, as shown by the smaller peak to peak separation.

Fig. 2f shows the cyclic voltammograms in a narrower potential window of 0 to $0.5 \mathrm{~V}$. The voltammograms exhibit a much more rectangular shape, and essentially the specific areal capacitance can be enhanced by loading a larger amount of $\mathrm{CoF}_{2}$ via the growth of thicker layers. Note that the gravimetric capacitance calculated from voltammograms at $50 \mathrm{mV} \mathrm{s}^{-1}$ exhibit values of $147 \pm 5 \mathrm{~F} \mathrm{~g}^{-1}$ for all layer thicknesses of 0.8 , 3.5 and $7 \mu \mathrm{m}$. Therefore, no extra capacitance was derived from bulk cobalt foil. The advantage of $\mathrm{CoF}_{2}$ in comparison to $\mathrm{Co}_{3} \mathrm{O}_{4}$ is that for the thicker layer, the redox kinetics is still reasonably fast (see Fig. S3a and b, ESI $\dagger$ ). This enables the charging/ discharging of a higher amount of material per unit time and per unit electrode area. This feature is particularly useful in the case of an application as a microsize capacitor where the areal capacitance becomes important.

Fig. 3a shows the cyclic voltammograms as a function of scan rate for $\mathrm{a} \sim 7 \mu \mathrm{m}$ thick $\mathrm{CoF}_{2}$ layer. The data show that the voltammograms retain nearly rectangular CV loops up to a scan 
(a)
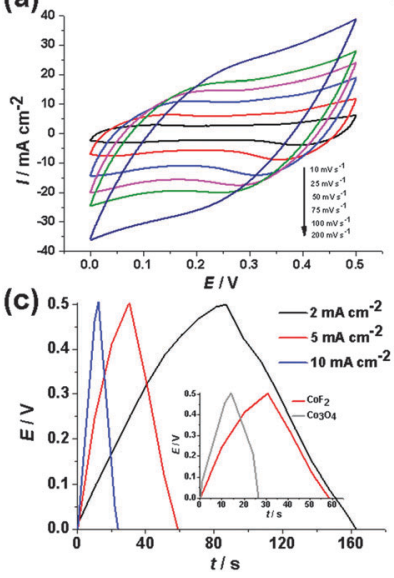

(b) ${ }_{300}$

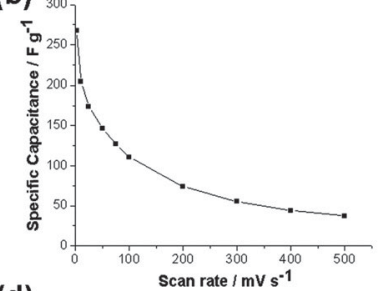

(d)

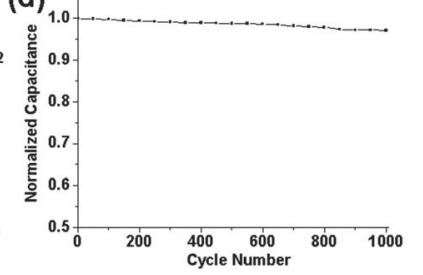

Fig. 3 (a) Cyclic voltammograms of a $7 \mu \mathrm{m}$ thick $\mathrm{CoF}_{2}$ sample under different scan rates in $1.0 \mathrm{M} \mathrm{KOH}$ solution. (b) Plot of specific capacitance vs. scan rate. (c) Galvanostatic charge-discharge curves of the $\mathrm{CoF}_{2}$ nanoporous layers with different current densities. (d) Capacitance retention test of the $\mathrm{CoF}_{2}$ nanoporous layers under $1000 \mathrm{CVs}$ cycles at a scan rate of $100 \mathrm{mV} \mathrm{s}^{-1}$.

rate of $100 \mathrm{mV} \mathrm{s}^{-1}$, which are characteristic for supercapacitors with an excellent capacitance behavior and a low contact resistance. Essentially, each curve consists of a capacitive current, and the curves at different scan rates show no peaks; this indicates that the electrodes are charged and discharged at a pseudoconstant rate over the complete voltammetric cycle in this potential range. Variations in the specific capacitance values as a function of scan rates are presented in Fig. 3b. At a scan rate of $2 \mathrm{mV} \mathrm{s}^{-1}$, a specific capacitance of $268 \mathrm{~F} \mathrm{~g}^{-1}$ was obtained. The specific capacitance value from this work $\left(204 \mathrm{~F} \mathrm{~g}^{-1}\right.$ at $10 \mathrm{mV} \mathrm{s}^{-1}$ ) is almost on par with the recent work on vertically aligned graphene nanosheets $\left(230 \mathrm{~F} \mathrm{~g}^{-1}\right.$ at $\left.10 \mathrm{mV} \mathrm{s}^{-1}\right)$ - also measured in a three electrode cell configuration. ${ }^{18}$ As the scan rate increases, the specific capacitance of the electrode decreases, as higher scan rate prevents the access of ions to the entire pores of the electrode - the movement of ions is limited by their slow diffusion, and therefore only the outer surface of the electrode can be utilized for charge storage.

The galvanostatic charge-discharge curves of $\mathrm{CoF}_{2}$ collected at various current densities are shown in Fig. 3c. The specific capacitance calculated from the curves is $272 \mathrm{~F} \mathrm{~g}^{-1}, 261 \mathrm{~F} \mathrm{~g}^{-1}$ and $223 \mathrm{~F} \mathrm{~g}^{-1}$ at a current density of 2,5 and $10 \mathrm{~mA} \mathrm{~cm} \mathrm{~cm}^{-2}$, respectively. In general, at lower current densities, the specific capacitance decreases with the increase in discharge current density, and the phenomenon is the same as the one observed in the case of increasing scan rate. It should be noted that still around $82 \%$ initial capacitance is retained (please compare to $272 \mathrm{~F} \mathrm{~g}^{-1}$ at $2 \mathrm{~mA} \mathrm{~cm}^{-2}$ ), even when the current density increases as high as $10 \mathrm{~mA} \mathrm{~cm} \mathrm{~cm}^{-2}$, reflecting the good highrate performance of the material.

Interestingly, the charge-discharge curves of $\mathrm{CoF}_{2}$ nanoporous samples are highly symmetric, with only a very slight curvature. This indicates that the supercapacitor behavior is mainly influenced by EDLCs contribution along with the redox reactions (pseudocapacitors). At $2 \mathrm{~mA} \mathrm{~cm}^{-2}$, the charge-discharge profile

shows only a small IR drop $(0.08 \mathrm{~V})$. At $5 \mathrm{~mA} \mathrm{~cm}{ }^{-2}$, almost a negligible IR drop is seen for $\mathrm{CoF}_{2}$ samples; however $\mathrm{Co}_{3} \mathrm{O}_{4}$ samples with the same thickness, as shown in the inset of Fig. 3c, show a significant IR drop of $0.3 \mathrm{~V}$. This reflects the better electrical conductivity of the $\mathrm{CoF}_{2}$ samples, with a much lower contact resistance. The cycling stability is tested at a scan rate of $100 \mathrm{mV} \mathrm{s}^{-1}$ for 1000 continuous voltammetric cycles. One can see that the $\mathrm{CoF}_{2}$ sample shows a good retention of $97 \%$ of the initial capacitance (Fig. 3d). To obtain conclusive information on the layer's long-term stability, significantly extended voltammetric cycling will however be required. Under the present experimental conditions, SEM analysis indicates that the aligned channel structure is maintained, although the discussed flakelike morphological changes occur under electrochemical oxidation in the $\mathrm{KOH}$ electrolyte as presented in Fig. 2a-d. Continued voltammetric cycling of a $7 \mu \mathrm{m}$ thick $\mathrm{Co}_{3} \mathrm{O}_{4}$ shows an increasing capacitance (see Fig. S4, ESI $\dagger$ ) presumably because of better ion transport and penetration into the thick crystalline $\mathrm{Co}_{3} \mathrm{O}_{4}$ layer for longer cycling.

The enhanced electrochemical capacitive performance of $\mathrm{CoF}_{2}$ can be attributed to three major factors: firstly, a faster redox kinetics that facilitates the transport of charge carriers that leads to the higher capacitance, secondly, the mixed crystalline and amorphous nature of $\mathrm{CoF}_{2}$ (as shown in the XRD) that increases the density of hydroxyl groups on the cobalt surface, thereby enhancing the pseudocapacitance and thirdly, the intrinsic properties of $\mathrm{CoF}_{2}$ with well-defined two-electron transfer process, as multiple oxidation states are beneficial for pseudocapacitor applications.

In summary, we have shown that an aligned $\mathrm{CoF}_{2}$ channel structure synthesized via anodic growth exhibits excellent supercapacitor properties. The distinct advantage of $\mathrm{CoF}_{2}$ is that it involves multiple electron transfer from $\mathrm{Co}$ (II) to $\mathrm{Co}$ (Iv) that provides multiple oxidation states with a more rectangular pseudocapacitive behavior that results in a high specific capacitance and a good performance of high charging-discharging rates. The electrode shows a good capacitance retention from 1000 voltammetric cycles. The present work thus suggests that fluoride based cobalt materials can be considered promising for supercapacitor applications.

This work was supported by grants from the DFG and the DFG cluster of excellence "Engineering of Advanced Materials" (EAM). The authors would like to acknowledge Helga Hildebrand for XPS measurements. A part of the present work was carried out using a facility in the Research Center for Ultra-High Voltage Electron Microscopy, Osaka University.

\section{Notes and references}

1 J. R. Miller and P. Simon, Science, 2008, 321, 651.

2 J. Bae, M. K. Song, Y. J. Park, J. M. Kim, M. Liu and Z. L. Wang, Angew. Chem., Int. Ed., 2011, 50, 1683.

3 B. E. Conway, Electrochemical Supercapacitors: Scientific Fundamentals and Technological Applications, Kluwer, Dordrecht, The Netherlands, 1999.

4 L. B. Hua, J. W. Choi, Y. Yang, S. Jeong, F. L. Mantia, L. F. Cui and Y. Cui, Proc. Natl. Acad. Sci. U. S. A., 2009, 106, 21490.

5 G. Wang, Y. Ling, F. Qian, X. Yang, X. X. Liu and Y. Li, J. Power Sources, 2011, 196, 5209.

6 M. Kaempgen, C. K. Chan, J. Ma, Y. Cui and G. Gruner, Nano Lett., 2009, 9, 1872. 
7 X. H. Lu, D. Z. Zheng, T. Zhai, Z. Q. Liu, Y. Y. Huang, S. L. Xie and Y. X. Tong, Energy Environ. Sci., 2011, 4, 2915.

8 H. Xia, J. K. Feng, H. L. Wang, M. O. Lai and L. Lu, J. Power Sources, 2010, 195, 4410.

9 L.-Q. Mai, F. Yang, Y.-L. Zhao, X. Xu, L. Xu and Y.-Z. Luo, Nat. Commun., 2011, 2, 381.

10 S. Trasatti and G. Buzzanca, J. Electroanal. Chem., 1971, 29, Appendix 1.

11 W. Sugimoto, H. Iwata, Y. Yasunaga, Y. Murakami and Y. Takasu, Angew. Chem., Int. Ed., 2003, 42, 4092.

12 R. B. Rakhi, W. Chen, D. Cha and H. N. Alshareef, Nano Lett., 2012, 12, 2559.
13 C.-Y. Lee, K. Lee and P. Schmuki, Angew. Chem., Int. Ed., 2013, 52, 2077.

14 P. Roy, S. Berger and P. Schmuki, Angew. Chem., Int. Ed., 2011, 123, 2956.

15 V. P. Parkhutik and V. I. Shershulsky, J. Phys. D: Appl. Phys., 1992, $25,1258$.

16 K. R. Hebert, S. P. Albu, I. Paramasivam and P. Schmuki, Nat. Mater., $2012,11,162$.

17 Y. Yang, S. P. Albu, D. Kim and P. Schmuki, Angew. Chem., Int. Ed., 2011, 50, 9071.

18 D. H. Seo, Z. J. Han, S. Kumar and K. Ostrikov, Adv. Energy Mater., 2013, 3, 1316. 